\title{
Decreased Monosynaptic Sensory Input to an Identified Motoneuron Is Associated with Steroid-mediated Dendritic Regression during Metamorphosis in Manduca sexta
}

\author{
Laura C. Streichert and Janis C. Weeks \\ Institute of Neuroscience, University of Oregon, Eugene, Oregon 97403-1254
}

The proleg withdrawal reflex of Manduca sexta larvae is mediated by mono- and polysynaptic connections from afferents innervating mechanosensory planta hairs (PHs) to ipsilateral proleg retractor motoneurons. During the larvalpupal transformation, a rise in ecdysteroids causes the dendrites of proleg motoneurons to regress and, concurrently, the proleg withdrawal reflex is lost. The objective of this study was to identify synaptic correlates of dendritic regression that contributed to this behavioral change. The accessory planta retractor (APR) motoneuron regresses during the larval-pupal transformation and dies in a segment-specific pattern after pupation. The compound excitatory postsynaptic potential (cEPSP) evoked in APR by electrically stimulating the proleg sensory nerve decreased in amplitude during the larval-pupal transformation. The developmental decrease in CEPSP amplitude, the extent of APR's regression, and several of APR's Intrinsic electrical properties were similar in segments in which the motoneuron was fated to live or die. In heterochronic mosaic pupae bearing retained larval PH afferents, APR's dendritic regression was associated with decreases in both the mean amplitude of monosynaptic EPSPs produced by PH afferents and the proportion of PH afferents that produced detectable EPSPs. These changes appeared due to a developmental decrease in the size of the synaptic currents produced by PH afferents. Evidence was also obtained suggesting that PH afferents do not regress at pupation. These findings support the hypothesis that the ecdysteroid-mediated regression of proleg motoneuron dendrites disconnects them from sensory inputs and, thereby, contributes to the elimination of the proleg withdrawal reflex.

[Key words: insect, afferent, mechanoreceptors, monosynaptlc, sensory neuron, motoneuron, regression, steroid hormone, moth]

Hormones can dramatically affect neuronal structure, as exemplified by the effects of gonadal steroids on the dendritic structure of neurons during development and in adulthood (reviewed

\footnotetext{
Received Apr. 22, 1994; revised Aug. 8, 1994; accepted Aug. 15, 1994

We thank Seana $K$. Davidson for camera lucida drawings, Dr. Emma R. Wood for statistical assistance, and Dr. Gwen A. Jacobs, Dr. Richard B. Levine, Dr. Peter B. Sargent, and other colleagues for insightful comments on the manuscript. This work was supported by NRSA Grant NS08966 to L.C.S. and NIH Grants NS23208 and NS01473, and an NSF Presidential Young Investigator Award, to J.C.W.

Correspondence should be addressed to Janis C. Weeks, Institute of Neuroscience, 1254 University of Oregon, Eugene, OR 97103-1254.

Copyright $C 1995$ Society for Neuroscience $0270-6474 / 95 / 151484-12 \$ 05.00 / 0$
}

in McEwen, 1991; Breedlove, 1992). In many cases, natural or experimentally induced hormonal changes that alter an animal's behavior also produce concomitant neuroanatomical changes, suggesting that the hormonal effects on neuronal structure underlie the behavioral changes. However, few studies have used electrophysiological techniques to test the links between hormonally induced neuroanatomical and behavioral changes.

In the hawkmoth, Manduca sexta, ecdysteroids induce the restructuring of motoneuron arbors during metamorphosis (reviewed in Weeks and Levine, 1992). These dendritic changes presumably contribute to the reconfiguration of neural circuits underlying behavior, but synaptic and behavioral correlates of dendritic remodeling have been identified in only a few cases (Levine and Truman, 1982; Jacobs and Weeks, 1990). One behavioral change associated with metamorphosis is the loss of the proleg withdrawal reflex. In Manduca larvae, tactile stimulation of mechanosensory planta hairs (PHs) on the proleg tip activates a withdrawal reflex mediated by mono- and polysynaptic connections from PH sensory neurons to the motoneurons that innervate ipsilateral proleg retractor muscles (Weeks and Jacobs, 1987; Trimmer and Weeks, 1991). During the larval-pupal transformation, a rise in ecdysteroids causes the dendrites of proleg motoneurons to regress (Weeks, 1987; Weeks et al., 1992), and concurrently the withdrawal reflex is lost. Jacobs and Weeks (1990) found that dendritic regression of the principal planta retractor (PPR) motoneuron was associated with a decrease in the amplitude of the compound excitatory postsynaptic potential (cEPSP) evoked by electrically stimulating the proleg sensory nerve to activate the $\mathrm{PH}$ afferents. Using hormonal manipulations to generate heterochronic mosaic pupae with retained larval $\mathrm{PH}$ afferents and regressed proleg motoneurons, they found that developmental changes in the motoneurons appeared sufficient to produce the decrease in cEPSP amplitude. These findings led to the hypothesis that ecdysteroid-mediated regression of proleg motoneuron dendrites causes synaptic connections from the $\mathrm{PH}$ afferents to be weakened and/or eliminated, resulting in the loss of the behavior (Jacobs and Weeks, 1990).

The present study tested this hypothesis by measuring the amplitude of unitary, monosynaptic EPSPs evoked in the accessory planta retractor (APR) motoneuron by $\mathrm{PH}$ afferents in normal and heterochronic mosaic insects. We found that dendritic regression was associated with decreases in both the mean amplitude of unitary EPSPs produced in APR by PH afferents, and the proportion of PHs that produced detectable EPSPs. These changes appeared to result from a developmental decrease in the synaptic currents produced by the afferents. APR dies in a segment-specific pattern after pupation (Weeks and Ernst- 
Utzschneider, 1989), which allowed us to also examine whether several of APR's properties, including dendritic regression and the decrease in afferent input, were correlated with its subsequent fate. No correlations were found, consistent with other studies suggesting that motoneuron regression and death are independently regulated (Weeks, 1987; Weeks et al., 1992). Finally, we obtained evidence suggesting that $\mathrm{PH}$ afferents do not regress during normal development. These findings support the tested hypothesis and successfully demonstrated an electrophysiological link between hormonally mediated neuroanatomical and behavioral changes.

Some data have appeared in abstract form (Streichert et al., 1992).

\section{Materials and Methods}

Insects. Manduca sexta were raised in a colony maintained on a 17L: $7 \mathrm{D}$ photoperiod and $27: 25^{\circ} \mathrm{C}$ thermoperiod. Larvae were reared individually in cups and fed an artificial diet (modified from Bell and Joachim, 1976). Larvae were staged as day L0 on the first day of the 5th (final) larval instar (Fig. 1A). At the initiation of metamorphosis (wandering, day W0), larvae were placed into individual chambers bored in wooden blocks. Pupae were staged as day $\mathrm{PO}$ on the day of larval-pupal ecdysis. Other times of development were designated as days after $\mathrm{L} 0$, W0, or P0 (e.g., L3 is the third day after day L0). Insects of both sexes were used.

Preparations. Insects were anesthetized with $\mathrm{CO}_{2}$ gas before dissection and all experiments were conducted at room temperature (23$25^{\circ} \mathrm{C}$ ). Preparations were bathed in saline containing $140 \mathrm{~mm} \mathrm{NaCl}, 5$ $\mathrm{mm} \mathrm{KCl}, 4 \mathrm{~mm} \mathrm{CaCl}_{2}, 28 \mathrm{~mm}$ glucose, $5 \mathrm{~mm}$ HEPES, at pH 7.4 (Trimmer and Weeks, 1989).

For the isolated ganglion preparation, an abdominal ganglion was removed from the body and pinned ventral side up in a Sylgard-lined petri dish. The lateral branch of the ventral nerve $\left(\mathrm{VN}_{\mathrm{L}}\right)$, which contains the proleg motor axons (Weeks and Truman, 1984), and another branch of the $\mathrm{VN}$, designated $\mathrm{VN}_{\mathrm{AAb} 3}$, which carries the axons of $\mathrm{PH}$ afferents (Jacobs and Weeks, 1990; Trimmer and Weeks, 1993), were left long for extracellular recording or stimulation (see below). The attachedproleg preparation (see Fig. 4A; Weeks and Jacobs, 1987) consisted of the distal tip (planta) of a proleg connected to the ganglion of the same segment via $\mathrm{VN}_{\mathrm{AAbr} 3}$. The planta was pinned to a Sylgard-lined recording dish with the PHs down and the inner epidermal surface facing up, to expose the PH sockets (Trimmer and Weeks, 1989). The arrangement of the sockets corresponded to the pattern of PIIs described by Peterson and Weeks (1988). In day L3 larvae, the first (distal-most) row of 1020 sockets is approximately $3.5-4.5 \mathrm{~mm}$ long. These sockets are adjoined by several rows of irregularly spaced sockets on the more proximal planta. For experiments in which individual sockets were identified and electrically stimulated (see below), a map of the first few rows of sockets was drawn using a drawing tube on a Wild binocular microscope. Typically, the largest one or two hairs in the PH array are located in the middle of the first row (Weeks and Jacobs, 1987; Peterson and Weeks, 1988). We have designated these the $\alpha$-PHs. Before pinning the preparation to the dish, the $\alpha-\mathrm{PHs}$ were identified and cut short so their corresponding sockets could be located. The socket corresponding to an $\alpha-\mathrm{PH}$ was identified in the first row of sockets and used as a midpoint to divide the array into three regions. The boundaries of the middle region were set $0.5 \mathrm{~mm}$ anterior and $0.5 \mathrm{~mm}$ posterior to the $\alpha$-PH (if there were two $\alpha-\mathrm{PHs}$, then the midpoint was set between the two). The anterior and posterior regions included the PHs anterior and posterior, respectively, to these boundaries. Thus, the length of the middle region of the PH array was $1.0 \mathrm{~mm}$, and the length of the anterior and posterior regions was $1.25-2.25 \mathrm{~mm}$; differences in the length of the anterior and posterior regions resulted from variation in the number of small hairs at the edges of the array.

Electrophysiological techniques. Differential extracellular nerve recordings were made using glass-tipped suction electrodes and amplified using high-gain AC-coupled preamplifiers (Grass Instrument Company, Quincy, MA). Intracellular recording electrodes were pulled from glass capillary tubing and filled with $2 \mathrm{~m} \mathrm{~K}$-acetate and $10 \mathrm{mM} \mathrm{KCl}$ (resistance 30-50 M $\Omega$ ). Intracellular recordings were amplified with a Dagan 8800 amplifier (Dagan Instruments, Minneapolis, MN) uscd either in bridge or single electrode current clamp (SEC) mode (see below). Data were stored on magnetic tape using an eight-channel recorder (Vetter Instruments, Rebersberg, PA) and later digitized ( $10 \mathrm{kHz})$ and analyzed on a PC equipped with data analysis software (RC Electronics, Santa Barbara, CA). For some experiments, recordings were made directly to the PC for off-line analysis.

All intracellular recordings were made from the APR motoneurons, which innervate the accessory planta retractor muscle (APRM) of the larval proleg (Weeks and Truman, 1984). Ganglia in larval abdominal segments three through six (A3-A6, Fig. 1A) each contain two pairs of APR motoneurons. Members of each pair of APRs are indistinguishable by a number of anatomical and physiological criteria and share the innervation of the ipsilateral APRM of the same segment (Weeks and Ernst Utzschneider, 1989; Sandstrom and Weeks, 1991; Sandstrom, 1993). Only APRs in segments A3 and A6 were used in this study. Ganglia were treated for $2-3 \mathrm{~min}$ with $3 \%$ collagenase-dispase and desheathed (Weeks and Jacobs, 1987). APR somata were impaled under visual guidance using transmitted dark-field illumination. Because the APRs are the only motoneurons with somata on the ventral ganglionic surface and axons in the ipsilateral VN (Weeks and Truman, 1984), they were identified unambiguously by the presence of extracellular spikes recorded in the $\mathrm{VN}$ or $\mathrm{VN}_{\mathrm{L}}$. To ensure the accuracy of voltage measurements made while passing current, cEPSPs and EPSPs were recorded in bridge mode after balancing the electrode in SEC mode. Injected current was used to hyperpolarize APR's membrane potential to

$-60 \mathrm{mV}$ from its normal value of approximately $-48 \mathrm{mV}$ (Table 1) when recording synaptic events, to eliminate spontaneous firing and standardize the recording conditions (e.g., Jacobs and Weeks, 1990).

The input resistance $\left(R_{\mathrm{in}}\right)$ of the APRs was determined by injecting a series of hyperpolarizing pulses $(0$ to $-2 \mathrm{nA}, 600 \mathrm{msec}, 0.1 \mathrm{~Hz})$ into the soma and measuring the steady-state voltage in SEC mode. $R_{\text {in }}$ was calculated from the slope of the line regressed through the current/ voltage $(I / V)$ relationship, which was linear in this range of currents (Sandstrom, 1993). The time constant $(\tau)$ of the APRs was determined as the time between the onset of the hyperpolarizing pulse and the point at which the voltage reached $63.2 \%$ (i.e., I $-1 / \mathrm{e}$ ) of its steady-state value. The value of $\tau$ for each APR was determined from the mean of $\geq 3$ hyperpolarizing pulses. We did not attempt to determine the relative contribution of somatic and dendritic membrane to the values of $R_{\text {in }}$ and $\tau$ measured in this study.

Recording of cEPSPs. cEPSPs were evoked in APRs by applying voltage pulses $(0.1-0.3 \mathrm{msec}, 0.03 \mathrm{~Hz})$ to the ipsilateral $\mathrm{VN}_{\mathrm{AAbr}}$ via a glass-tipped suction electrode connected to a Grass S88 stimulator. An extracellular electrode placed en passant on the proximal VN monitored the size of the evoked sensory volley and stimulation intensity was adjusted to activate all of the PH sensory axons (e.g., Jacobs and Weeks, 1990). cEPSP amplitude was measured as the difference between APR's membrane potential immediately prior to the onset of the synaptic depolarization and at the peak of the depolarization, disregarding action potentials. Peak cEPSP amplitude was calculated as the mean of $\geq 3$ trials for each APR.

To determine the relative contribution of mono- and polysynaptic components to cEPSPs, responses were recorded in normal saline and then in a "high-divalent" saline containing elevated concentrations (20 $\mathrm{mm}$ ) of $\mathrm{MgCl}_{2}$ and $\mathrm{CaCl}_{2}$ ( $\mathrm{NaCl}$ was reduced to maintain correct osmolarity; Jacobs and Weeks, 1990). The intensity of $\mathrm{VN}_{\mathrm{AAbr} 3}$ stimulation was readjusted to maintain maximal sensory neuron activation. Because high-divalent saline raises spike threshold and thereby suppresses polysynaptic pathways (Berry and Pentreath, 1976), cEPSPs recorded in this saline presumably reflected the monosynaptic component of the response. High-divalent saline was perfused into the recording dish until the response stabilized $(-3-5 \mathrm{~min})$ and then $\geq 3$ stimulus trials were averaged. Data were accepted only when cEPSP amplitude recovered to approximately the control value after replacing high-divalent saline with normal saline (see Results).

Recording of unitary EPSPs. Unitary EPSPs evoked in APR by individual PH afferents were recorded using the attached-proleg preparation. Each $\mathrm{PH}$ is innervated by a single sensory neuron whose cell body is subjacent to the $\mathrm{PH}$ and whose axon projects into the ganglion of the same segment (Peterson and Weeks, 1988). Individual PH sensory neurons were stimulated at $1.0 \mathrm{~Hz}$ with voltage pulses $(0.2 \mathrm{msec}$ duration) delivered via a glass suction electrode applied to the hair socket (see Fig. 4A; Trimmer and Weeks, 1989, 1991). At this stimulation frequency, the amplitude of evoked EPSPs is stable indefinitely (Trimmer and Wecks, 1991). Afferent spikes werc monitored by an extracellular electrode placed en passant on the proximal VN, and the 


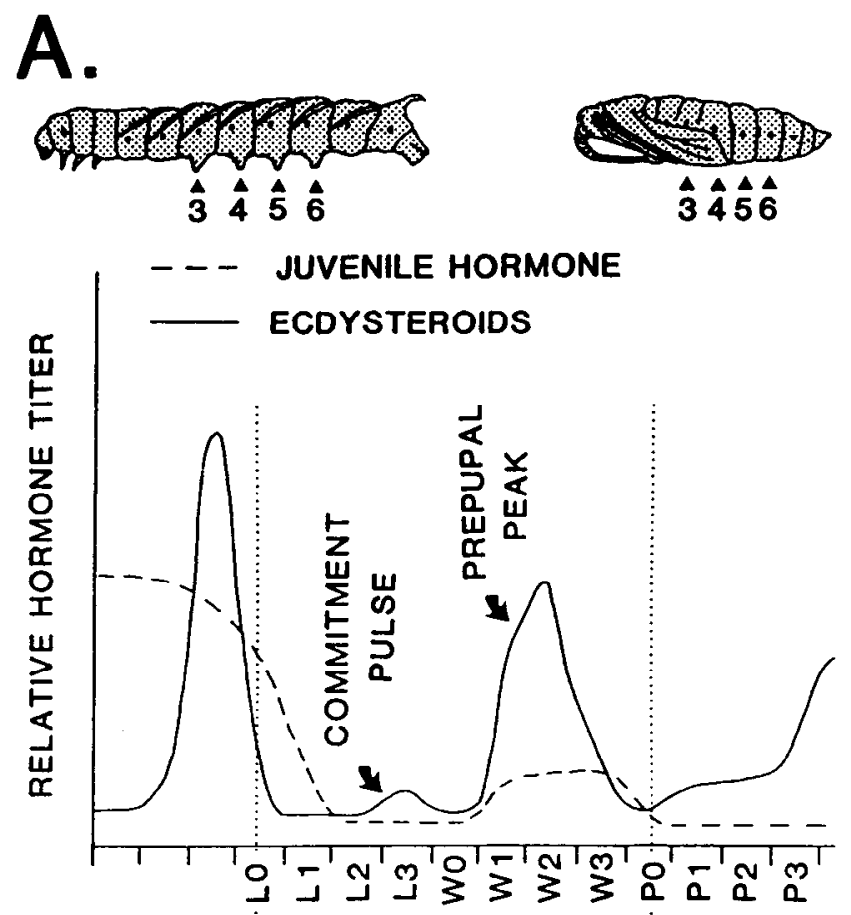

4th LARVA $\dot{\mathrm{E}}$

5th LARVA

$\dot{E}$

C.

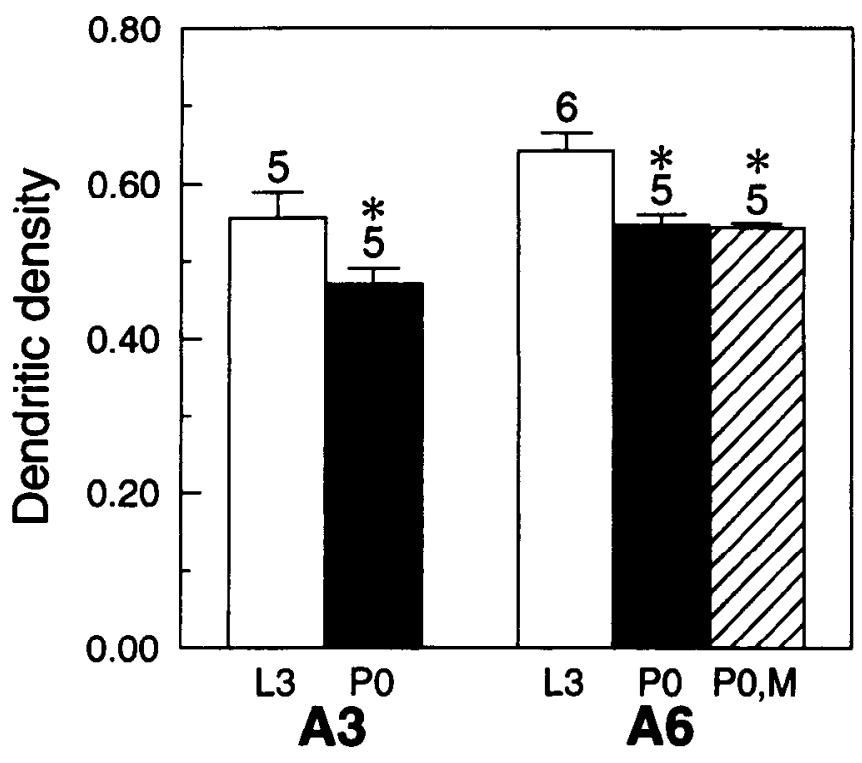

B.
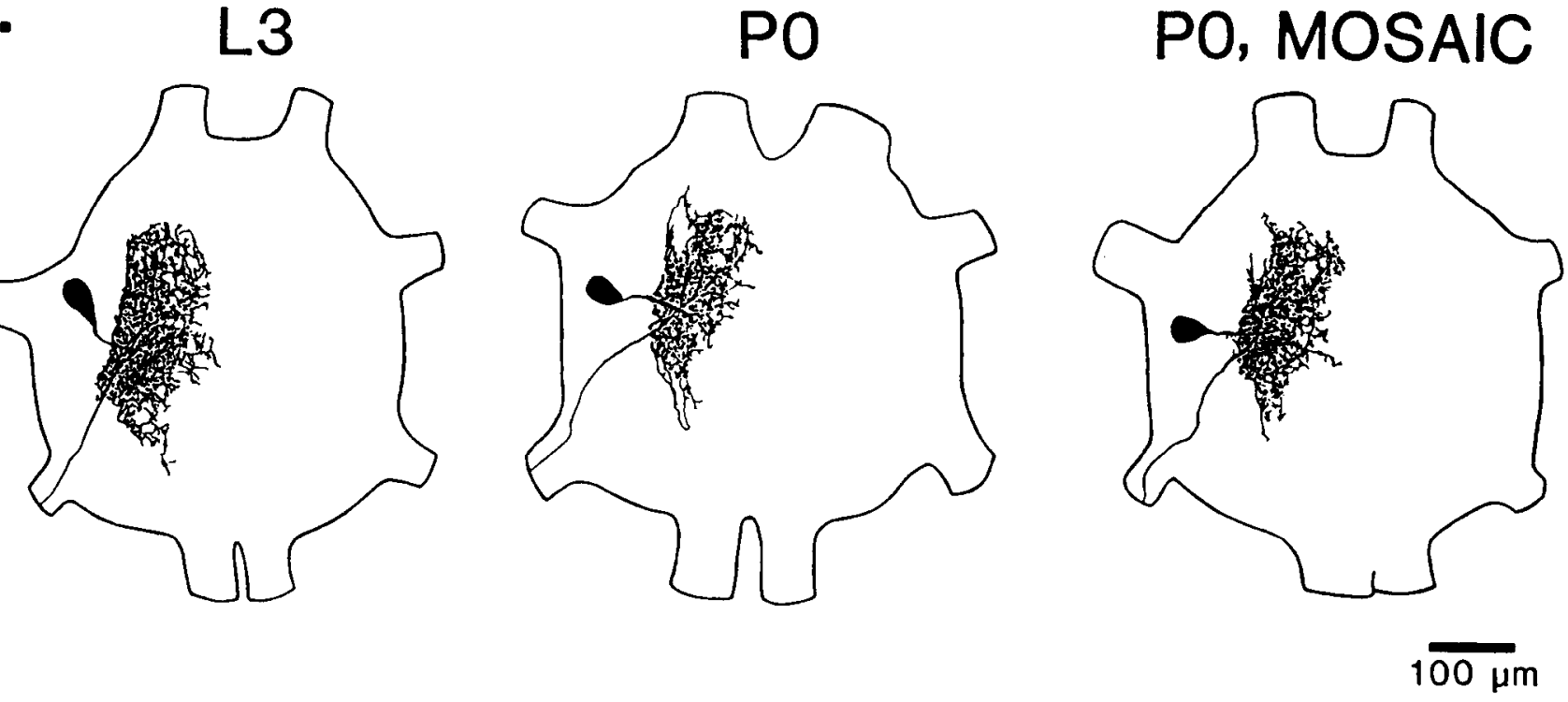

Figure 1. A, Time line of endocrine events during the larval-pupal transformation, showing changes in the relative hemolymph titers of ecdysteroids (solid line) and juvenile hormone (dashed line; modified from Bollenbacher et al., 1981 and Riddiford, 1985). Days are labeled as described in Materials and Methods. Dotted lines mark ecdyses $(E)$ from the 4th to 5th larval instar and from the larva to pupa. Drawings of a larva and pupa are shown with segments $A 3-A 6$ indicated. $B$, Dendritic regression of APR during the larval-pupal transformation. APRs were stained by intracellular iontophoresis of cobalt. In this and subsequent figures, ganglia are shown in dorsal view with anterior up. Camera lucida drawings of wholemounted ganglia are shown in segment A6 from normal insects on days L3 and P0, and a heterochronic mosaic insect on day P0. APR's dendritic arbor was less extensive in both normal and mosaic day P0 ganglia than in day L3 ganglia. $C$, Dendritic density measurements were analyzed on day L3 (open bars) and day P0 (filled bars) in segments A3 and A6. APR's mean dendritic density was significantly reduced (*) on day P0 as compared to day L3, and this decrease was similar in segments A3 and A6. In segment A6, APR's mean dendritic density in mosaic hemisegments $\left(P O, M\right.$; hatched bar) was significantly reduced from the value on day L3 $\left(^{*}\right)$. The mean dendritic density of APRs in normal and mosaic hemisegments on day PO did not differ significantly. Data are presented as mean \pm SEM, with $n$ indicated above each bar. See Results for statistical comparisons. 
Table 1. Intrinsic electrical properties of the APRs

L3

P0

P0 mosaic

Segment A3

Membrane potential (mV)

Input resistance $(\mathrm{M} \Omega)$

Time constant (msec)

Segment A6

Membrane potential $(\mathrm{mV})$

Input resistance $(\mathrm{M} \Omega)$

Time constant (msec)
$-48.6 \pm 0.8(18)$
$45.2 \pm 1.8(18)$
$26.5 \pm 1.4(16)$
$-48.0 \pm 0.4(51)$
$43.4 \pm 1.5(43)$
$24.9 \pm 1.0(39)$

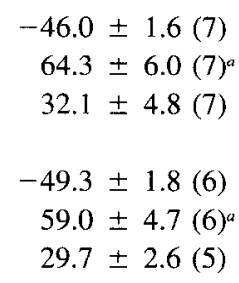

$\begin{aligned}-47.8 & \pm 0.8(25) \\ 56.8 & \pm 2.4(23)^{b} \\ 24.1 & \pm 2.2(18)\end{aligned}$

All values are mean \pm SEM $(n)$. See Results for statistical comparisons.

"Significant difference between values on day P0 and day L3.

${ }^{h}$ Significant difference between value for day PO mosaic and day L3.

stimulus voltage applied to the socket was adjusted until it was suprathreshold for a single sensory neuron. Intracellular recordings were made from APRs ipsilateral to the attached proleg. All EPSP measurements were made from the computer-generated average of $\geq 20$ trials. Peak amplitude of unitary EPSPs was determined as for cEPSPs; the detection limit was $0.05 \mathrm{mV}$. The time-to-peak of each EPSP was measured as the interval between the start of the upward deflection of the EPSP and the time of maximum depolarization. EPSP delay was measured as the latency from the socket stimulus to the onset of the upward deflection of the EPSP. When sensory neuron stimulation failed to produce a detectable EPSP in APR, the response was measured as the difference between APR's membrane potential immediately before the socket stimulus and that measured $15 \mathrm{msec}$ after the stimulus; this was the approximate latency from the stimulus to the peak depolarization for detectable EPSPs (see Results).

Histological techniques. The structures of motoneurons and sensory neurons were visualized by cobalt staining. APRs were impaled with an electrode containing $100 \mathrm{~mm}$ cobalt chloride, and cobalt was iontophoresed using $600 \mathrm{msec}$ depolarizing pulses of $10-20 \mathrm{nA}$ at $1 \mathrm{~Hz}$ for 5-15 min. The cobalt was precipitated in saline saturated with $\mathrm{H}_{2} \mathrm{~S}$ gas, fixed in Carnoy's fixative, and silver intensified (modified from Bacon and Altman, 1977). Ganglia were dehydrated in a graded EtOH series, cleared in methyl salicylate, and whole mounted in Canada Balsam. Canera lucida drawings were made with a drawing tube on a Leitz Laborlux D microscope at a final magnification of $540 \times$. The extent of APR's dendritic arbor was determined by a "dendritic density" score that measured the proportion of the neuropil occupied by dendritic processes (Weeks and Truman, 1985; Miles and Weeks, 1991). Only ganglia in which staining of APR's processes appeared uniform and complete were analyzed. The terminal arbors of PH sensory neurons within the CNS were stained by anterograde filling of the $\mathrm{VN}_{\mathrm{AAbr} 3}$ with $2 \%$ $\mathrm{CoCl}_{2}$ (Jacobs and Weeks, 1990) and processed and drawn as described above. Morphometric parameters of individual afferents were measured from camera lucida drawings using a digitizing tablet and PC equipped with drawing software (SIGMASCAN, Jandel Scientific, Corte Madera, CA).

Heterochronic mosaics. During metamorphosis, larval tissues become committed to pupal differentiation in response to a small elevation of ecdysteroids (the commitment pulse) on day L3 that occurs in the absence of juvenile hormone (JH; Fig. 1A). A larger prepupal peak of ecdysteroids that spans days W1 to P0 causes development of the pupa (Fig. 1A; Riddiford, 1985; Bollenbacher, 1988). Exposure of larval tissue to $\mathrm{JH}$ during the commitment pulse blocks the ability to produce pupal tissues during the prepupal peak (Levine et al., 1986; Weeks and Truman, 1986). Topical treatment of a proleg with a JH analog (methoprene; a gift from Zoecon Corp., Palo Alto, CA) during the commitment pulse produces heterochronic mosaic pupae that retain the treated proleg and its PH array (Jacobs and Weeks, 1990). In the present study, methoprene was dissolved at $1 \mathrm{mg} / \mathrm{ml}$ in dental wax, applied to a proleg early on day $\mathrm{L} 2$, and removed $2 \mathrm{~d}$ later. Because the developing pupal wing extends over the site of the proleg in segment A3 (Fig. 1A), heterochronic mosaics were generated in segment $A 6$ only. The size of the patch of retained proleg epidermis, and the number of PHs, varied among treated insects. Mosaics were included in the study if the retained proleg had $\geq 45$ PHs (i.e., $\geq 75 \%$ of the number of PHs present on untreated prolegs in segment A6 on day L3; $60 \pm 1, n=5$ ), and if their arrangement appeared normal. The APRM in the treated hemisegments, which would normally degenerate at pupation (Sandstrom, 1993), was not preserved by methoprene treatment. Developmental rates were similar in methoprene-treated, wax only-treated, and untreated insects. All heterochronic mosaic insects were studied on the day of larval-pupal ecdysis and, for brevity, the term "day P0 mosaics" is used henceforth to refer to heterochronic mosaic hemisegments on day P0. All experiments utilized APRs ipsilateral to the retained larval proleg.

Statistics. Data are presented as mean \pm standard error of the mean (SFM). A Student's two-tailed $t$ test, a chi-squared test, or a one- or two-way analysis of variance (ANOVA) followed by post hoc twotailed $t$ tests with Bonferroni-adjusted significance levels were used for statistical comparisons, as appropriate (BMDP Statistical Software, Inc., Los Angeles, CA). Significance was assumed when $p<0.05$.

\section{Results}

Dendritic regression of $A P R$ in normal and heterochronic mosaic insects

During the larval-pupal transformation, the dendritic arbors of the APRs regress, as is typical for proleg motoneurons (Weeks and Truman, 1984, 1985; Weeks and Ernst-Utzschneider, 1989; Sandstrom, 1993). However, the APRs are unique among these motoneurons in displaying segment-specific fates; in response to the prepupal peak of ecdysteroids (Fig. 1A), APRs in segments A5 and A6 die on days P1-P2, whereas the homologous APRs in segments A3 and A4 survive this period, are respecified for new pupal or adult functions, and die after adult emergence (Weeks and Ernst-Utzschneider, 1989; Weeks et al., 1992; Sandstrom, 1993). To determine whether the extent of dendritic regression was correlated with the subsequent fate of an APR, developmental changes in the APRs' dendritic arbors were measured in segments A3 and A6, in which the APRs are fated to survive or die, respectively, after pupation.

Figure $1 B$ illustrates the regression of APR's dendritic arbor in segment A6 during the larval-pupal transformation. Dendritic density measurements on days L3 and P0 (Fig. 1C) were analyzed by a two-way ANOVA for segment and developmental stage. There was a significant main effect of stage $[F(1,17)=$ $14.11, p<0.01$ ], indicating that APR's dendritic density decreased significantly between days L3 and P0. Although there was a significant main effect of segment $[F(1,17)=11.21, p<$ $0.01]$, there was no significant two-way interaction, indicating that the extent of dendritic regression between days L3 and PO was similar for APRs in segments A3 and A6 (a decrease in dendritic density of $15.2 \%$ and $14.9 \%$, respectively). Thus, at the level of resolution provided by our morphometric technique, the extent of dendritic regression was similar in APRs destined to live or die. 
A.

L3

Po

A3
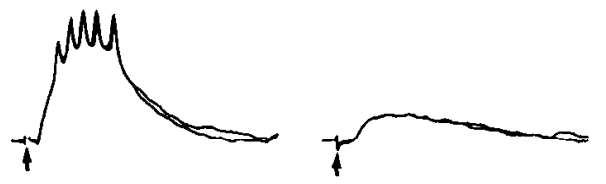

A6

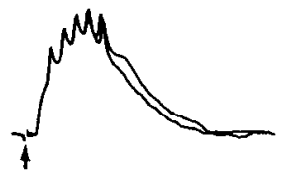

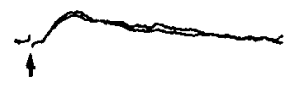

PO, MOSAIC

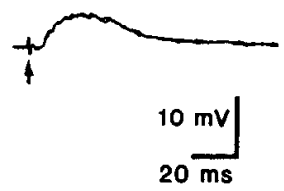

B.

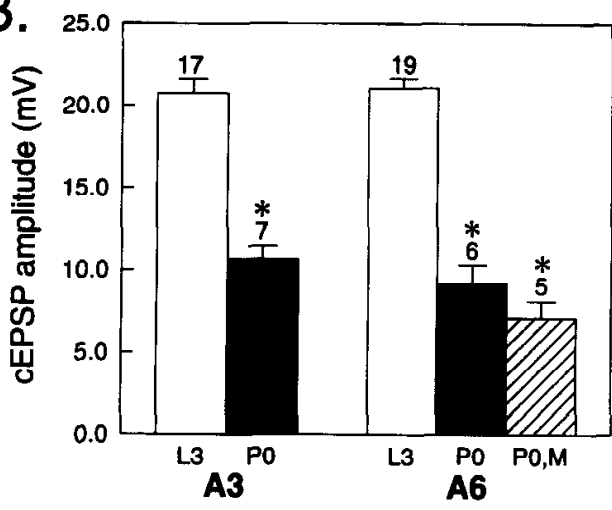

Figure 2. The developmental decrease in cEPSP amplitude was uncorrelated with APR's fate or the developmental status of the PH afferents. A, Single supramaximal electrical stimuli applied to $\mathrm{VN}_{\mathrm{AAbr} \text {. }}$ (stimulus artifacts marked by arrows) evoked cEPSPS in APR that were smaller on day P0 than on day L3. On both days, cEPSPS appeared qualitatively similar in segments A3 and A6. cEPSPs evoked in APR in a mosaic hemisegment on day P0 are shown from segment A6 only, and resembled those produced in normal day P0 hemisegments. APR's membrane potential was hyperpolarized to $-60 \mathrm{mV}$ during all recordings, and each panel shows two superimposed traces. $B$, Mean cEPSP amplitude was significantly reduced (*) on day $\mathrm{P0}$ (filled bars) as compared to day L3 (open bars) in segments A3 and A6. In segment A6, mean cEPSP amplitude in day P0 mosaics ( $P O, M$; hatched bar) was also significantly smaller than the value on day L3 $\left({ }^{*}\right)$. Mean cEPSP values in normal and mosaic hemisegments on day P0 did not differ significantly. Data are presented as mean \pm SEM, with $n$ indicated above each bar. See Results for statistical comparisons.

A major source of monosynaptic excitatory input to larval proleg motoneurons is provided by ipsilateral PH afferents (Jacobs and Weeks, 1990). During the degeneration of the proleg epidermis between days L3 and P0 (Jacobs and Weeks, 1990), the extent of the central termination field of the $\mathrm{PH}$ afferents decreases (see below), which could potentially provide a signal for APR regression. This possibility was tested by examining APR's regression in day $\mathrm{P} 0$ mosaics that bore a retained larval proleg and its associated $\mathrm{PH}$ array on segment A6 (see Materials and Methods). PH sensory neurons associated with a retained proleg in mosaics survive, continue to project normally into the CNS, and maintain their somatotopic organization in the neuropil (Jacobs and Weeks, 1990). A further motivation for making these measurements was that many of the electrophysiological experiments in this study utilized mosaics (see below), and it was important to verify that the APRs in mosaic hemisegments regressed normally. APR's dendritic structure in day $\mathrm{PO}$ mosaics appeared indistinguishable from that of APRs in normal day P0 pupae (Fig. $1 B$ ). Furthermore, a one-way ANOVA comparing APR's dendritic density on day L3 and on day P0 in normal and mosaic hemiscgments (Fig. $1 C$ ) revealcd a significant main effect of stage $[F(2,13)=11.36, p<0.01]$, but there was no significant difference between mean dendritic density in the normal and mosaic day P0 hemisegments (post hoc $t$ test). Thus, APR's dendritic regression appeared to occur normally in mosaic hemisegments despite the retention of larval PH afferents.

\section{Measurements of cEPSPs and intrinsic electrical properties of $A P R$ in normal and heterochronic mosaic insects}

Jacobs and Weeks (1990) showed that the amplitude of the cEPSP evoked in PPR by synchronously activating the PH afferents with electrical stimulation of the proleg sensory nerve $\left(\mathrm{VN}_{\mathrm{AAbr} 3}\right)$ decreased significantly as the motoneuron regressed. We repeated this experiment for the APRs, and compared findings in segments $\mathrm{A} 3$ and $\mathrm{A} 6$ in which APR fate differed. On day L3, single supramaximal shocks to $\mathrm{VN}_{\mathrm{AAbr} 3}$ evoked cEPSPs in APRs in A3 and A6 that were suprathreshold and evoked action potentials (Fig. $2 A$ ). On day P0, VNAAbr3 stimulation produced cEPSPs in A3 and A6 that were smaller than on day L3 and subthreshold (Fig. 2A). Measurements of mean cEPSP amplitude from day L3 and day PO insects (Fig. $2 B$ ) were analyzed by a two-way ANOVA for segment and developmental stage. Therc was a significant main effect of stage $[F(1,45)=$ $127.15, p<0.001]$, but no significant effect of segment and no significant interaction. Therefore, the reduction in mean cEPSP amplitude between days L3 and P0 was similar in segments A3 and A6, in which APRs have different fates.

We also measured cEPSPs in day $P 0$ mosaics bearing a retained proleg on segment A6 (Fig. 2A,B). A one-way ANOVA comparing data from day $\mathrm{L} 3$, day $\mathrm{PO}$, and day $\mathrm{P0}$ mosaics in segment A6 revealed a significant main effect of stage $[F(2,27)$ $=80.27, p<0.001]$. Post hoc $t$ tests indicated that mean cEPSP amplitude in day $P 0$ mosaics was significantly reduced as compared to day L3 $[t(27)=10.33, p<0.001]$, and cEPSP amplitude on day $\mathrm{PO}$ in normal and mosaic hemisegments did not differ significantly. This finding suggested that developmental changes in the APRs' properties, independent of the status of the $\mathrm{PH}$ afferents, were sufficient to produce the developmental decrease in cEPSP amplitude.

Developmental changes in the APRs that could have produced decreased cEPSP amplitude included dendritic regression (Fig. 1) and alterations in the motoneurons' intrinsic electrical properties. To examine the latter possibility, we measured the APRs' resting membrane potential $\left(V_{m}\right)$, input resistance $\left(R_{\text {in }}\right)$, and time constant ( $\tau$ ) on days $\mathrm{L} 3$ and $\mathrm{PO}$ in segments $\mathrm{A} 3$ and A6, and on day P0 in mosaic segment A6 (Table 1). For each electrical property, comparisons of segment and stage were done with a two-way ANOVA. For $V_{m}$ and $\tau$, there were no significant main effects and no significant interactions, indicating that the mean values were similar in all groups. In contrast, $R_{\text {in }}$ increased significantly between days L3 and P0 (Table 1). This was indicated by a significant main effect for stage, in which the mean values on day $\mathrm{P} 0$ were significantly greater than on day $\mathrm{L} 3[F(1,70)=$ 29.13, $p<0.001]$. There was no significant main effect of segment, indicating that $R_{\mathrm{in}}$ increased similarly in segments $\mathrm{A} 3$ and A6. $R_{\text {in }}$ measurements made in segment A6 on day L3, day P0, 
and in day PO mosaics were compared by a one-way ANOVA for stage followed by post hoc $t$ tests. The mean $R_{\text {in }}$ of APRs in day P0 mosaics was significantly greater than on day L3 [t(39) $=4.62, p<0.001]$, and $R_{\mathrm{in}}$ measured in the normal and mosaic day $\mathrm{P0}$ hemisegments did not differ significantly. The developmental increase in APR's $R_{\text {in }}$ may result from a decrease in membrane area during the regression of APR's dendritic arbor. Because an increase in $R_{\text {in }}$ would be expected to increase the size of the synaptic potential produced by a given synaptic current, the decrease in cEPSP amplitude observed between days L 3 and P0 (Fig. 2) may underestimate the actual developmental change in synaptic input.

\section{Developmental changes in monosynaptic EPSPs produced in} APR by $P H$ afferents

Previous experiments demonstrated that proleg motoneurons re ceive both mono- and polysynaptic input from ipsilateral $\mathrm{PH}$ sensory neurons (Weeks and Jacobs, 1987; Jacobs and Weeks, 1990). Because the focus of this study was on developmental changes in the monosynaptic connections from $\mathrm{PH}$ afferents to the APRs (see below), we investigated the relative contribution of mono- and polysynaptic pathways to the developmental decrease in cEPSP amplitude. $\mathrm{VN}_{\mathrm{AAbr} 3}$ was stimulated on days L3 and $\mathrm{P} 0$, and cEPSPs were recorded while bathing the ganglion in normal saline and then in saline containing elevated levels of $\mathrm{Ca}^{2+}$ and $\mathrm{Mg}^{2+}$ to suppress polysynaptic pathways (Fig. 3). The component of the cEPSP that persisted in high-divalent saline was presumed to be monosynaptic. Similar results were obtained in segments A3 and A6 (data not shown), so data from both segments were combined. A two-way ANOVA revealed significant main effects of developmental stage $[F(1,42)=55.44, p$ $<0.001]$ and saline type $[F(2,42)=20.83, p<0.001]$, and a significant two-way interaction $[F(2,42)=5.35, p<0.01]$. The mean amplitude of cEPSPs evoked in high-divalent saline was significantly smaller than the amplitude in normal saline both on days L3 and P0 [post hoc $t$ tests; L3, $t(19)=8.95, p<0.001$; $\mathrm{P} 0, t(5)=3.51, p<0.02]$. The cEPSP amplitude recovered when normal saline was restored, and there was no significant difference between normal and wash values on either day (post hoc $t$ tests). Mean cEPSP amplitude was significantly reduced on day $\mathrm{PO}$ as compared to day $\mathrm{L} 3$ under all saline conditions [post hoc $t$ tests; normal saline, $t(13)=7.96, p<0.001$; highdivalent saline, $t(6)=2.52, p<0.05$; wash, $t(8)=6.20, p<$ $0.001]$. The significant decrease in mean cEPSP amplitude measured in high-divalent saline between days L3 and P0 suggested that the presumed monosynaptic component of the response decreased during the larval-pupal transformation.

The developmental decrease in the amplitude of the presumed monosynaptic component of the cEPSP could have resulted from decreases in the amplitude of individual unitary EPSPs, a reduction in the proportion of sensory neurons that produced EPSPs, or a combination of both mechanisms. To examine developmental changes in synaptic transmission between individual PH sensory neurons and APR, the attached-proleg preparation (Fig. 4A) was used to stimulate individual $\mathrm{PH}$ sockets while recording EPSPs in APR. Recordings were made in segment A6 on day L3 and in day P0 mosaics. Heterochronic mosaics were used on day $\mathrm{P} 0$ because the proleg normally degenerates by day $P 0$ and individual hair sockets cannot be stimulated (Jacobs and Weeks, 1990). Furthermore, because APR's dendritic regression and the developmental decrease in cEPSP amplitude occurred similarly in mosaic and normal day P0 hemisegments (Figs. 1,

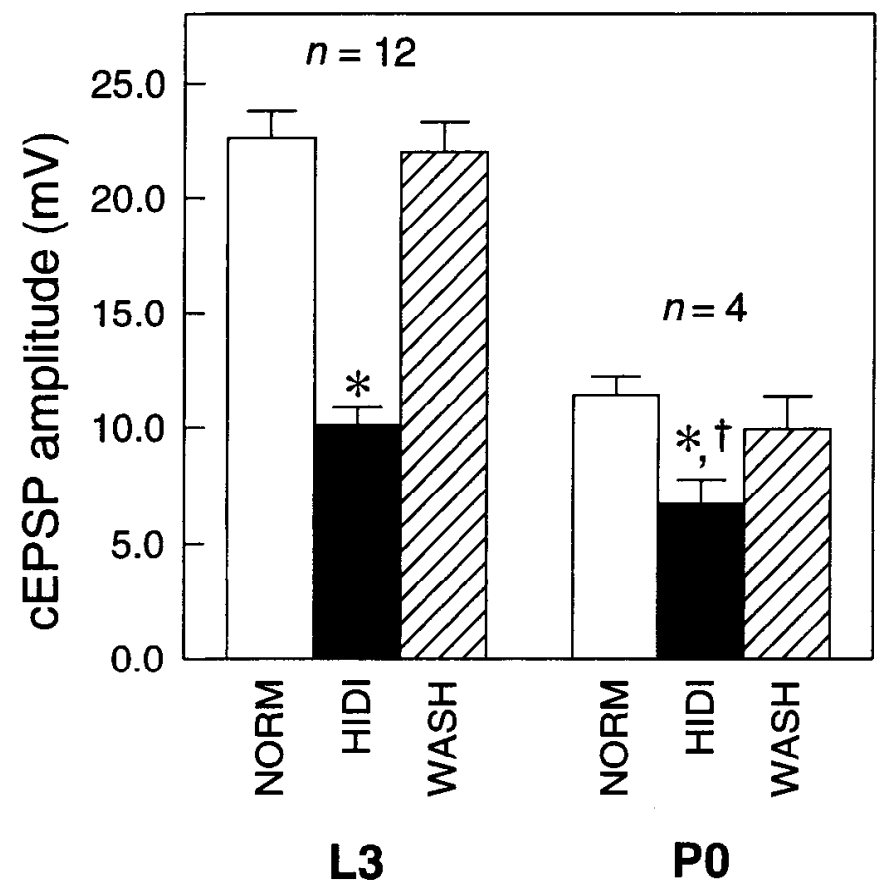

Figure 3. The presumed monosynaptic component of cEPSPs decreased during the larval-pupal transformation. The relative contribution of mono- and polysynaptic components to the cEPSP was determined by comparing the mean amplitude of cEPSPs evoked in normal saline (NORM; open bars) with that in high-divalent saline (HIDI; filled bars), which suppressed the polysynaptic component. On days L3 and P0, cEPSPs evoked in high-divalent saline were significantly smaller (*) than those evoked in normal saline. cEPSP amplitude recovered when normal saline was restored (WASH; hatched bars), and there was no significant difference between normal and wash values on either day L3 or P0. Mean cEPSP amplitude was significantly smaller on day P0 than that on day L3 under all saline conditions. The significant decrease in mean cEPSP amplitude in high-divalent saline between days L 3 and P0 ( $\dagger$ ) suggested that the presumed monosynaptic component of the response decreased during the larval-pupal transformation. In all cases, the normal, high-divalent, and wash values for cEPSP amplitude were repeated measurements from the same APRs; data from segments A3 and A6 were combined. APR's membrane potential was hyperpolarized to $-60 \mathrm{mV}$ during all recordings. Data are presented as mean \pm SEM, with $n$ for each day indicated above the bars. See Results for statistical comparisons.

2), differences between EPSPs recorded on days L3 and P0 were most likely due to changes in APR rather than in $\mathrm{PH}$ afferents (see Discussion).

Stimulation of individual PH sensory neurons on day L3 produced time-locked spikes in the VN and EPSPs in ipsilateral APRs (Fig. 4B). Consistent with previous studies (e.g., Weeks and Jacobs, 1987; Trimmer and Weeks, 1991), the afferentevoked EPSPs appeared monosynaptic by a number of criteria. The EPSPs followed PH afferent stimulation with a constant delay (9.6 $\pm 0.3 \mathrm{msec} ; n=29)$ and persisted without failure during rapid stimulation ( $>20 \mathrm{~Hz}$; data not shown). $\mathrm{PH}$ afferents also evoked unitary EPSPs in APR in day P0 mosaics (Fig. $4 C$ ). The voltage threshold for sensory neuron activation and the response latency were similar on day L3 and in day P0 mosaics (data not shown). Thus, afferent-evoked EPSPs in APR were qualitatively similar on day L3 and in day P0 mosaics.

In addition to making quantitative comparisons between EPSPs on day L3 and in day P0 mosaics, it was necessary to establish the relationship between $\mathrm{PH}$ position and EPSP amplitude. The axon terminals of PH sensory neurons arborize so- 


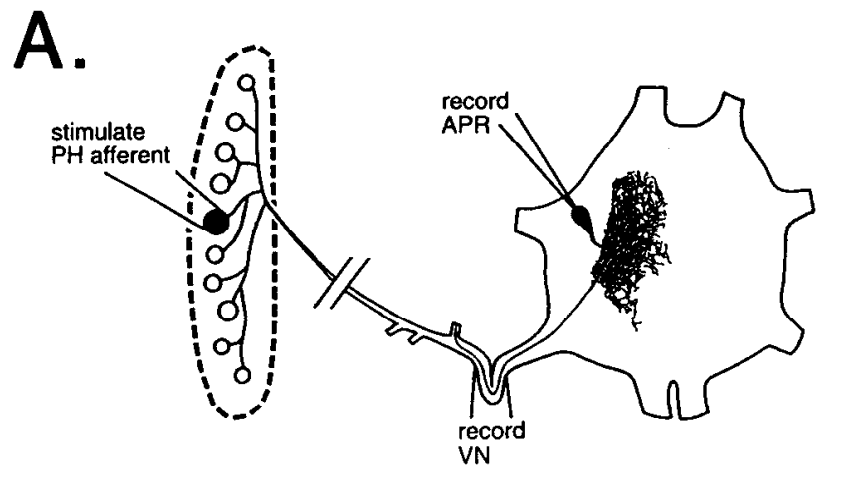

B. L3
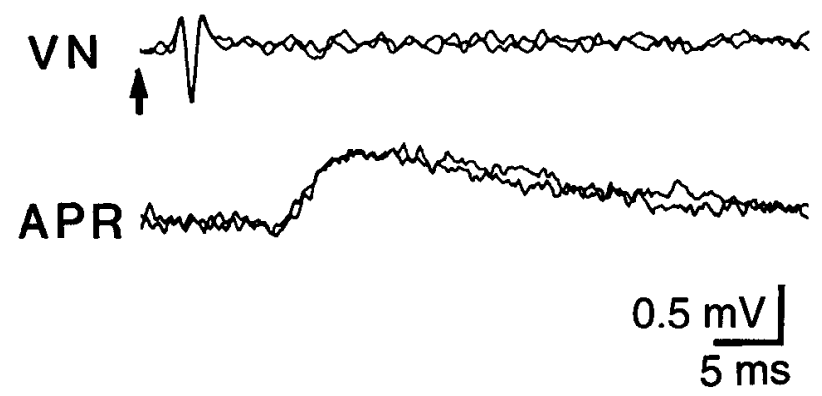

\section{PO, MOSAIC}
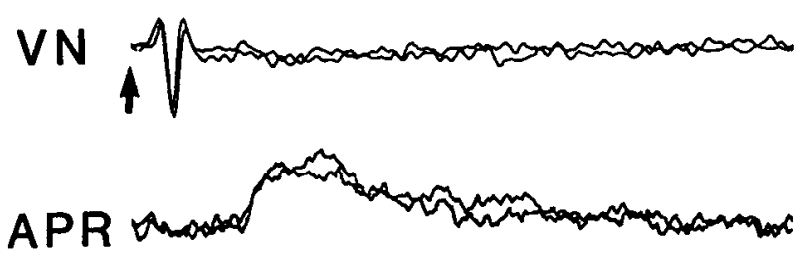

Figure 4. Stimulation of individual $\mathrm{PH}$ sensory neurons produced monosynaptic EPSPs in APR. A, The recording configuration of the attached-proleg preparation. To the left, the cpidermal surface of the planta is drawn schematically showing the sockets (circles) that correspond to the PHs in the distal-most row of the PH array. The proximal VN was monitored en passant to verify that each electrical stimulus applied to a socket produced a single sensory spike. Current pulses delivered to individual PH afferents (e.g., filled circle) were used to produce unitary EPSPs in APR. APR's membrane potential was hyperpolarized to $-60 \mathrm{mV}$ during all recordings. $\mathrm{PH}$ afferent stimulation evoked monosynaptic EPSPs in ipsilateral APRs on day L3 $(B)$ and in day P0 mosaics $(C)$. Each panel shows two superimposed, unaveraged trials. The upper trace in each panel shows sensory spikes recorded en passant in the ipsilateral VN in response to stimuli (arrows) applied to a middle socket in the first row of the $\mathrm{PH}$ array; lower traces show intracellular recordings from APR. Vertical scale bar refers to APR recording.

matotopically within the neuropil (Peterson and Weeks, 1988) and, in PPR, the size of the EPSP produced by an individual PH afferent is correlated with the position of the hair that it innervates; within the first (distal-most) row of hairs in the $\mathrm{PH}$ array, afferents innervating middle hairs (including the $\alpha$-PHs) produce the largest EPSPs, while afferents innervating anterior or posterior hairs produce smaller EPSPs or fail to produce detectable responses (Weeks and Jacobs, 1987; Levine and Weeks, 1989; Trimmer and Weeks, 1991).

To determine the pattern of sensory input to APR, we stim- ulated individual sensory neurons innervating PHs throughout the distal-most row of the $\mathrm{PH}$ array. Figure $5 \mathrm{~A}$ shows the arrangement of $\mathrm{PH}$ sockets in an attached-proleg preparation on day L3, and illustrates the characteristic differences in the amplitude of EPSPs evoked in an APR by PH afferents from different regions; the largest EPSP was produced by a middle afferent, while anterior and posterior afferents produced smaller EPSPs. In day P0 mosaics (Fig. 5B), the relationship between $\mathrm{PH}$ position and EPSP amplitude was similar to that on day L3. However, there was a lower incidence of detectable EPSPs (i.e., $\geq 0.05 \mathrm{mV}$ after signal averaging) in day PO mosaics, and the EPSPs that were detectable were smaller than those recorded on day L3.

Mean EPSP amplitude and the percentage of detectable EPSPs on day L3 and in day P0 mosaics are quantified in Figure 6. Figure $6 A$ shows the characteristic pattern of EPSP amplitudes evoked by $\mathrm{PHs}$ from different regions of the $\mathrm{PH}$ array. Comparison of EPSP amplitudes by a two-way ANOVA revealed a significant main effect of developmental stage $[F(1,45)=14.64$, $p<0.001]$ and a significant main effect of $\mathrm{PH}$ afferent position $[F(2,45)=23.86, p<0.001]$, but no significant interaction. The significant main effect of stage indicated that the mean amplitude of EPSPs produced by PHs in day P0 mosaics was significantly smaller than that of EPSPs produced by PHs in corresponding regions of the array on day L3. For instance, the mean amplitude of EPSPs produced by middle afferents was $36 \%$ smaller in day P0 mosaics than on day L3 (Fig. 6A). The mean amplitude of EPSPs produced in APR by anterior and posterior $\mathrm{PH}$ afferents was significantly smaller than that of EPSPs produced by middle PH afferents at both stages [post hoc $t$ tests; anterior afferents, $t(1)=6.02, p<0.001$; posterior afferents, $t(1)=6.08, p<0.001]$. There were no significant differences between the amplitude of EPSPs produced by anterior and posterior PHs on cither day (post hoc $t$ tests).

$\mathrm{PH}$ position was also related to the probability that an afferent produced a detectable synaptic response in APR (Fig. 6B). In all cases in which socket stimulation failed to produce a detectable EPSP in APR, a normal sensory spike was observed in VN (e.g., Fig. $4 C$ ) and other $\mathrm{PH}$ afferents in the same preparation produced detectable EPSPs (data not shown). Thus, failure to detect an EPSP appeared to result from the absence of a synaptic connection, or a synaptic current that was too small to produce a detectable voltage change in APR's cell body. Other than the $\alpha$-PHs, which had large sockets and invariably produced large EPSPs, the size of PH sockets was not correlated with the size of the synaptic effect in APR (data not shown). As shown in Figure $6 B$, middle $\mathrm{PH}$ afferents invariably produced detectable EPSPs in APR on day L3 and in day P0 mosaics. In contrast, anterior and posterior $\mathrm{PH}$ afferents sometimes failed to produce detectable responses (e.g., Fig. 5B), particularly in day $\mathrm{P0}$ mosaics. Specifically, the proportion of anterior and posterior $\mathrm{PH}$ afferents that elicited detectable EPSPs in APR in day P0 mosaics (4 of 11) was significantly smaller than the proportion of $\mathrm{PH}$ afferents from the same regions that produced detectable EPSPs on day L3 [16 of 19; chi-squared test, $\chi^{2}(1)=7.18, p$ $<0.01]$. Therefore, the developmental decrease in mean EPSP amplitude evoked by anterior and posterior $\mathrm{PH}$ afferents in day P0 mosaics (Fig. 6A) resulted from both a decrease in the mean amplitude of the detectable EPSPs (data not shown) and a decrease in the proportion of $\mathrm{PH}$ afferents that produced detectable EPSPs (Fig. 6B)

Differences in the amplitude of EPSPs evoked by individual 
A. L3
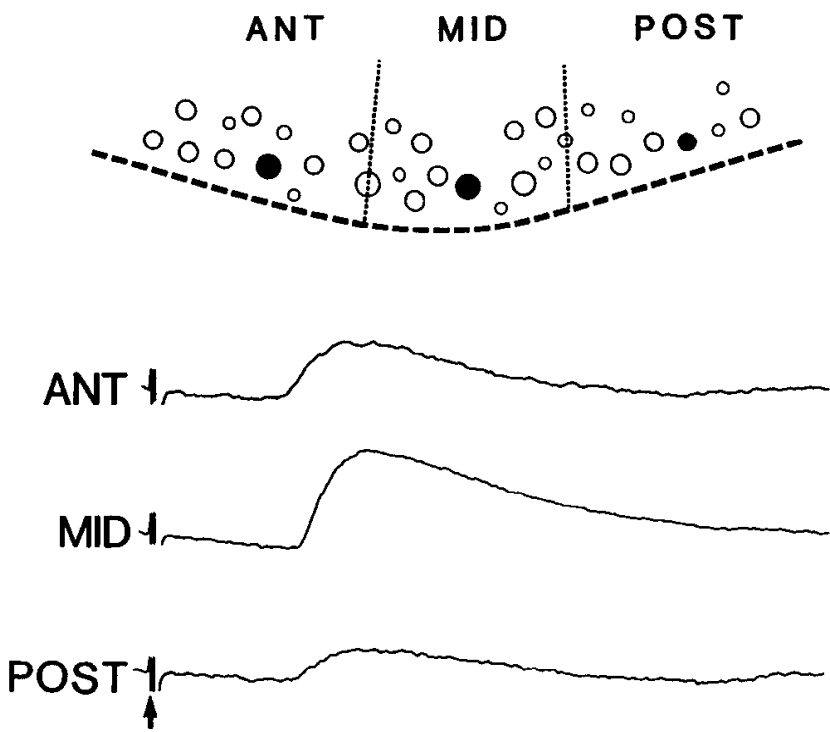

\section{B. PO, MOSAIC}

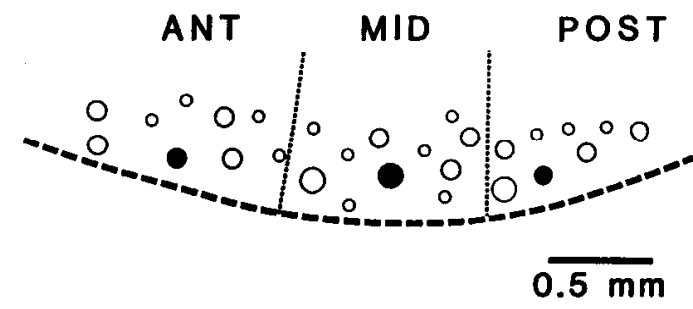

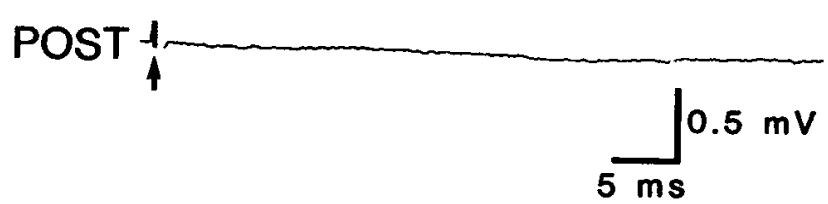

Figure 5. EPSP amplitude was related to PH position. Drawings above traces are schematic maps of sockets (circles) corresponding to individual $\mathrm{PHs}$ in the distal-most region of the PH array. Dashed line indicates the distal border of the PH array, epidermal side up; dotted lines demarcate anterior $(A N T)$, middle $(M I D)$, and posterior (POST) regions of the array. Filled circles indicate the sockets stimulated to produce the EPSPs shown below. Traces show averaged responses (20-60 trials) recorded in a single APR in response to stimulation of individual sensory neurons in different regions of the array on day L3 $(A)$ and in a day P0 mosaic $(B)$. All recordings were from segment A6. APR's membrane potential was hyperpolarized to $-60 \mathrm{mV}$ during all recordings. Arrows indicate stimulus artifacts.

PH sensory neurons could result from factors such as the amplitude of the synaptic current, the distance from the sites of synaptic contact to the recording site at APR's soma, or APR's intrinsic electrical properties. One feature of an EPSP that reflects the electrotonic distance from the synaptic contacts to the recording site is the time-to-peak, with more distant inputs generally having greater time-to-peak values (Rall et al., 1967; Jack et al., 1988). Figure 7 shows the mean time-to-peak values for detectable EPSPs evoked in APR by PH sensory neurons from the anterior, middle, and posterior regions of the $\mathrm{PH}$ array on day L3 and in day P0 mosaics. A two-way ANOVA for developmental stage and $\mathrm{PH}$ afferent position revealed no significant main effects and no significant interaction; thus, there were no significant differences in the time-to-peak of EPSPs at the two developmental stages or in different regions of the $\mathrm{PH}$ array. Although APR's $R_{\text {in }}$ increased significantly between days L3 and P0, $\tau$ did not change (Table 1). Therefore, differences in the size of EPSPs produced in APR by PH afferents from different regions of the $\mathrm{PH}$ array, and differences in EPSP amplitude in the two developmental stages (Figs. 5, 6A), appeared to result from differences in the amount of synaptic current rather than the electrotonic distance between the synaptic and recording sites or APR's intrinsic electrical properties.

\section{Morphology of individual PH afferents in larvae and pupae}

Dendritic regression and the developmental decrease in mean cEPSP amplitude were similar for APRs located in normal and mosaic day $\mathrm{P} 0$ hemisegments (Figs. $1 C, 2 B$ ), suggesting that developmental changes in $\mathrm{PH}$ afferents were unnecessary for these developmental changes in APRs. Nevertheless, it was of interest to determine the nature of the developmental changes normally exhibited by $\mathrm{PH}$ afferents. The central termination field of the $\mathrm{PH}$ afferent population within the ganglion of the same segment decreases in extent during the larval-pupal transformation (Fig. 8A; Jacobs and Weeks, 1990). This decrease involves the death of some PH afferents, and fails to occur in mosaic hemisegments (Jacobs and Weeks, 1990). The possibility that the decreased termination field might involve the regression of PH afferent arbors in addition to the death of afferents had not been previously addressed.

To test for $\mathrm{PH}$ afferent regression, we stained an individually identified PH afferent in normal insects on days L3 and P0, and analyzed its structure at both stages. The standard technique used to stain the central projection of a $\mathrm{PH}$ afferent-by cutting the hair and introducing cobalt-does not work in normal day $\mathrm{P0}$ insects because the afferent dendrites have disconnected from the hairs (Peterson and Weeks, 1988; Jacobs and Weeks, 1990). However, anterograde cobalt staining of $\mathrm{VN}_{\mathrm{AAtr} 3}$ revealed that a single $\mathrm{PH}$ afferent projected to the ganglion of the next posterior segment, allowing it to be viewed in isolation on days L3 and $\mathrm{P0}$ (Fig. 8A). This sensory neuron innervates an $\alpha-\mathrm{PH}$ (Peterson and Weeks, 1988).

When stimulated on day L3 in segment A5, the $\alpha$-PH afferent evoked an EPSP in the ipsilateral APR in segment A6 (Fig. 8B; $n=3$ preparations). Therefore, the axon terminals of the larval $\alpha-\mathrm{PH}$ afferent madc physiological contact with APR in the next posterior ganglion. For technical reasons, we could not test for this connection on day $\mathrm{PO}$. It is not possible to stimulate $\mathrm{PH}$ sockets in normal day P0 insects (see above), and although the experiment could have been carried out in day P0 mosaics, this would not have provided any information about the role of nor- 
A.

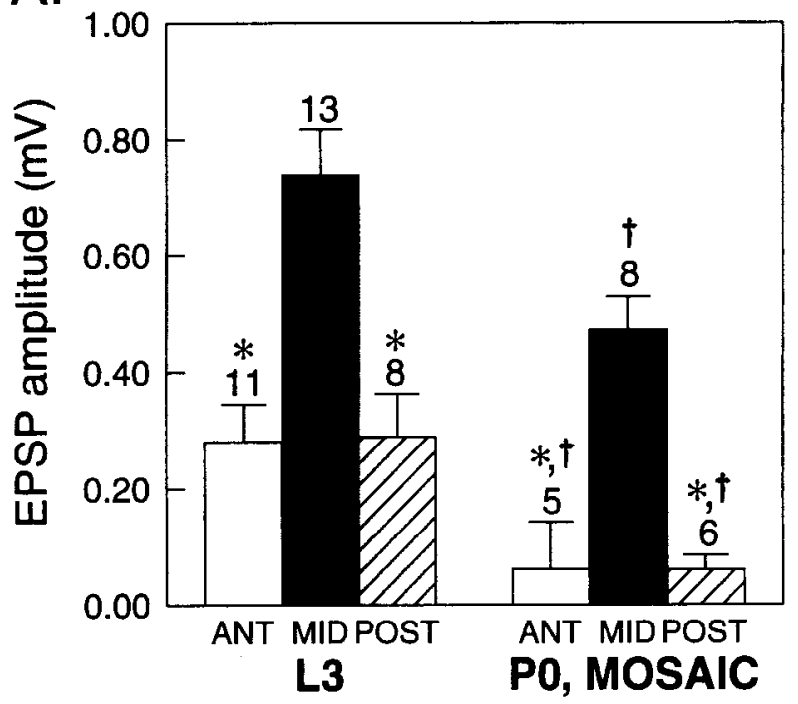

B.

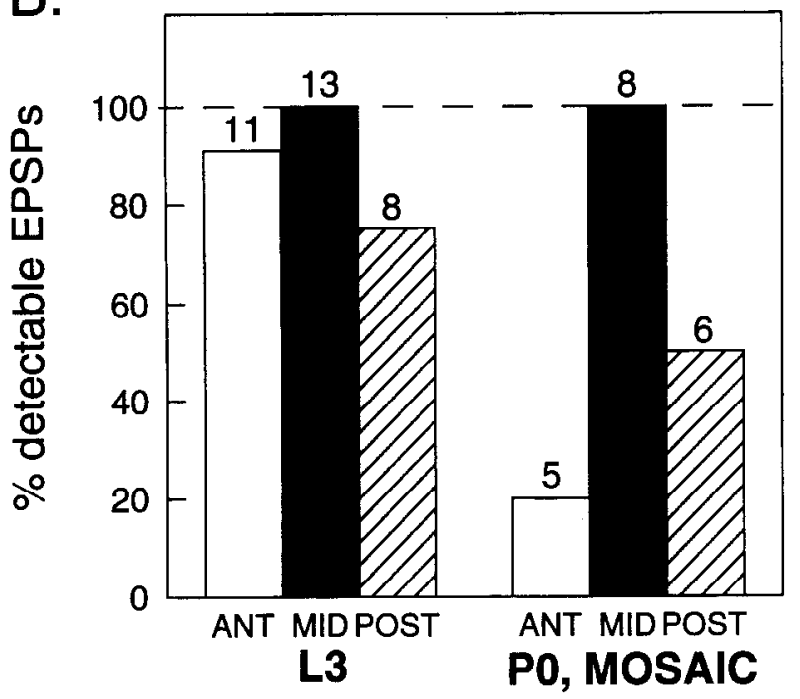

Figure 6. EPSP amplitude and the proportion of detectable EPSPs were related to $\mathrm{PH}$ position and developmental stage. The mean amplitude of signal-averaged unitary EPSPs evoked in APRs by PH afferents in the anterior (ANT; open bars), middle (MID; filled bars), and posterior (POST; hatched bars) regions of the first row of the PH array (see Fig. 5) were measured on day L3 and in day P0 mosaics. All recordings were from segment A6. Responses were classified as undetectable if their amplitude was $<0.05 \mathrm{mV}$. Mean amplitudes were computed from detectable and undetectable responses (and shown as mean \pm SEM). Data are from 19 preparations on day L3 and 10 preparations from day P0 mosaics, with $n$ (number of PH afferents tested) indicated above each bar. APR's membrane potential was hyperpolarized to -60 $\mathrm{mV}$ during all recordings. $A$, On day $\mathrm{L} 3$ and in day PO mosaics, the mean amplitude of EPSPs elicited in APR by anterior and posterior PH afferents was significantly smaller $\left({ }^{*}\right)$ than the mean amplitude of EPSPs produced by middle afferents. On both days, the mean amplitude of EPSPs generated by anterior and posterior PH afferents did not differ significantly. The mean amplitude of EPSPs produced by $\mathrm{PHs}$ in day PO mosaics was significantly smaller $(\dagger)$ than that of EPSPs produced by PHs in corresponding regions of the array on day L3. $B$, The proportions of PH afferents that produced detectable EPSPs $(\geq 0.05 \mathrm{mV})$ in APR are shown as a percentage of the total number tested. All middle PH afferents produced detectable EPSPs on both day L3 and in day P0 mosaics $(100 \%$, dashed line), whereas anterior and posterior $\mathrm{PH}$ afferents sometimes failed to produce detectable responses. In day P0 mosaics, a significantly smaller proportion of anterior and posterior $\mathbf{P H}$ afferents produced detectable EPSPs than on day L3. See Results for statistical comparisons.

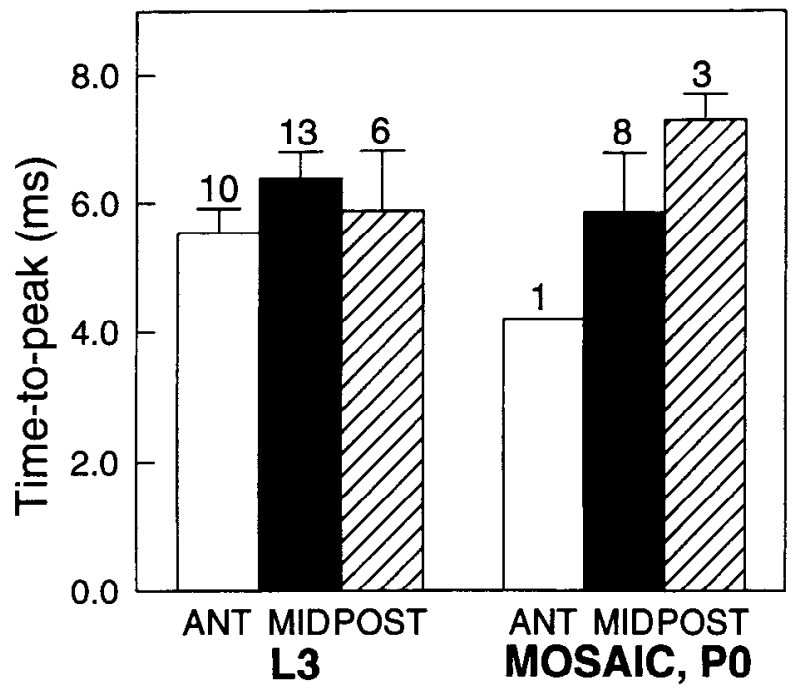

Figure 7. Unitary EPSPs generated by $\mathrm{PH}$ afferents in different regions of the PH array had similar time-to-peak values on day L3 and in day P0 mosaics. The time-to-peak of unitary EPSPs evoked in APRs by $\mathrm{PH}$ afferents in the anterior (ANT; open bars), middle (MID; filled bars) and posterior (POST; hatched bars) regions of the first row of the PH array were measured on day L3 and in day P0 mosaics. None of the values differed significantly. Data are from the detectable EPSPs in Figure 6, and are presented as mean \pm SEM, with $n$ indicated above each bar. See Results for statistical comparisons.

mal developmental changes in the afferents. We did attempt to activate the $\alpha-\mathrm{PH}$ afferent by stimulating $\mathrm{VN}_{\mathrm{AAbr} 3}$ in segment $\mathrm{A} 5$ and recording from APR in segment A6 in normal day P0 insects, but polysynaptic inputs activated by the stimulus made it impossible to distinguish a unitary EPSP in APR (data not shown).

The anatomy of the $\alpha-\mathrm{PH}$ afferent was compared on days L3 and $\mathrm{PO}$ by staining $\mathrm{VN}_{\mathrm{AAbr} 3}$ with cobalt in segment $\mathrm{A} 5$ and analyzing the axonal arbor of the $\alpha-\mathrm{PH}$ afferent on the ipsilateral side of segment A6 (Fig. 8A). Two morphometric parameters of the afferent arbor were measured: the number of branch points (Fig. 9A) and total branch length (Fig. 9B). There were no significant differences in either parameter on days L3 and PO $(t$ tests). This finding suggested that $\mathrm{PH}$ afferents alive on day $\mathrm{P0}$ had not undergone regression during the larval-pupal transformation.

\section{Discussion}

The present study demonstrated that dendritic regression of proleg motoneuron APR during the larval-pupal transformation (Fig. 1) was associated with a developmental decrease in the amplitude of the cEPSP evoked by stimulating the proleg sensory nerve, including a specific decrease in the presumed monosynaptic component (Figs. 2, 3). This decrease in the monosynaptic component resulted from a decrease in the mean amplitude of unitary, monosynaptic EPSPs evoked by stimulating individual PH afferents in all three regions (anterior, middle, and posterior) of the most distal row of the PH array (Figs. 5, 6). Both the proportion of afferents that produced detectable EPSPs and the amplitude of the detectable EPSPs were significantly reduced in day P0 mosaics as compared to day L3 (Fig. 6). These findings support the hypothesis that the ecdysteroid-mediated regression of proleg motoneuron dendrites disconnects them from $\mathrm{PH}$ afferent inputs and, thereby, contributes to the elimination 

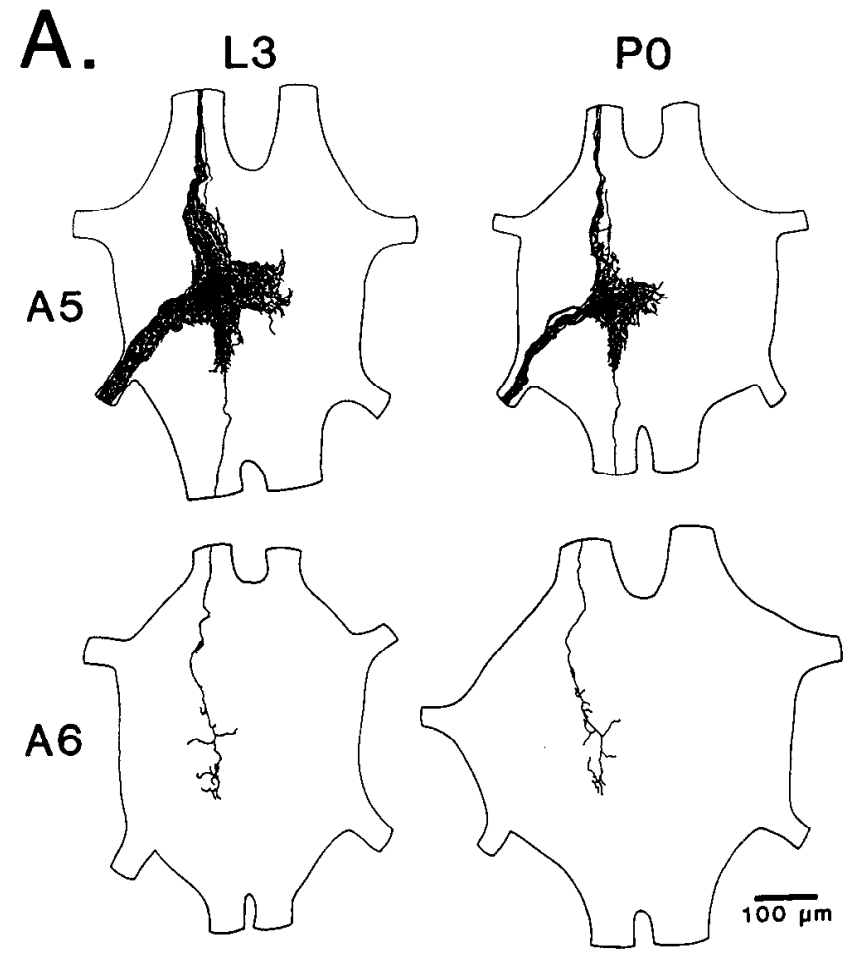

B.
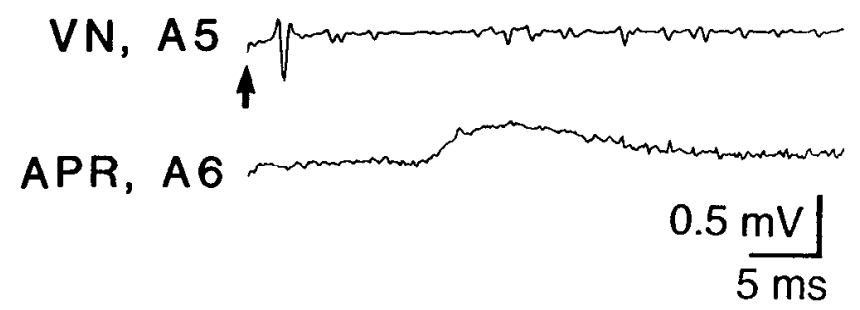

Figure 8. Central projections of PH afferents on days L3 and P0 in normal insects. $A$, Anterograde cobalt staining of the $\mathrm{VN}_{\mathrm{AAbr} 3}$ in segment A5 revealed a large termination field in ganglion A5 and the axon of a single $\alpha-\mathrm{PH}$ that projected to the ipsilateral hemineuropil in ganglion A6. Between days L3 and P0, the extent of the central termination field in A5 decreased. The axonal arbor of the $\alpha-\mathrm{PH}$ in ganglion $\mathrm{A} 6$ appeared similar on both days of development. $B$, On day $\mathrm{L} 3$, the $\alpha-\mathrm{PH}$ afferent from segment A5 produced an EPSP in APR in segment A6. The recording configuration was a modification of the attached-proleg preparation (Fig. $4 A$ ), consisting of a chain of two ganglia (A5 and A6) and a planta from segment A5. Stimulation of the socket of the $\alpha-P H$ in segment A5 (arrow) evoked a sensory spike recorded en passant in the ipsilateral VN in segment A5 (upper trace) and an EPSP in the ipsilateral APR in ganglion A6. Each trace is the average of 30 sweeps. APR's membrane potential was hyperpolarized to $-60 \mathrm{mV}$ during the recording. Vertical scale bar refers to APR recording.

of the proleg withdrawal reflex (Jacobs and Weeks, 1990). By this hypothesis, the regression of APR's dendrites causes some of the synaptic contacts between each PH afferent and APR to be lost; this would account for the finding that the large EPSPs produced by middle PHs on day L 3 became smaller on day P0, while the EPSPs from anterior and posterior PHs that were of modest size on day L3 became even smaller or fell below the detection limit on day P0 (Figs. 5, 6).

Mcasurements of APR's intrinsic electrical properties (Table 1), and the time-to-peak of the EPSPs (Fig. 7), suggested that
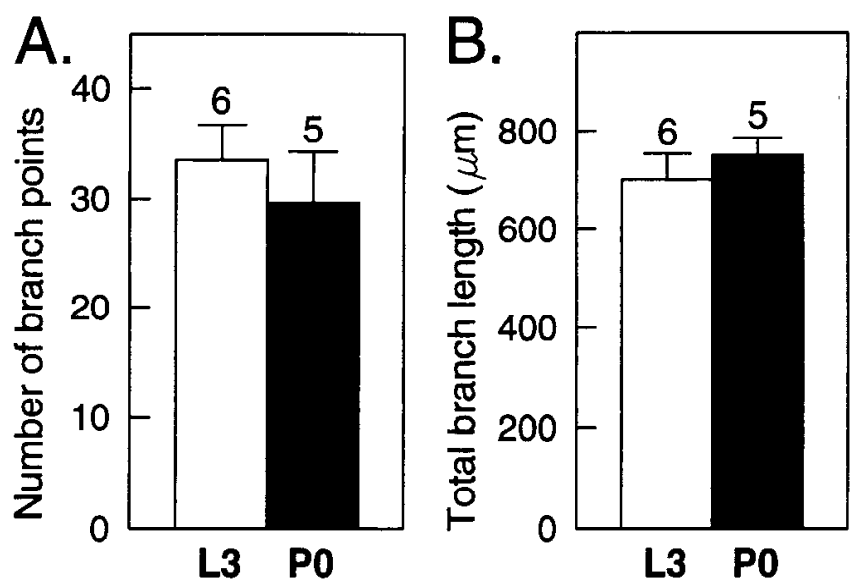

Figure 9. Comparison of the structure of the posterior axonal arbor of the $\alpha-\mathrm{PH}$ afferent on days L3 and P0. The axonal projection of the $\alpha-\mathrm{PH}$ afferent was visualized in ganglion $\mathrm{A} 6$ by anterograde staining of the $\mathrm{VN}_{\mathrm{AAbr} 3}$ in segment A5 (see Fig. 8A). Measurements were made from digitized camera lucida drawings of the cobalt-stained axonal arbor. The mean number of branch points $(A)$ and total branch length $(B)$ did not differ significantly on days L3 (open bars) and P0 (filled bars). Data are presented as mean \pm SEM, with $n$ indicated above each bar. See Results for statistical comparison.

the decreased EPSP amplitude resulted from a decrease in the amplitude of the synaptic current produced by individual $\mathrm{PH}$ afferents. The total synaptic current is the sum of the current produced at each synaptic contact between the pre- and postsynaptic neurons. Therefore, a developmental decrease in the number of synaptic contacts between $\mathrm{PH}$ afferents and APR could explain the decrease in synaptic current. A relationship between the number of synaptic contacts and the size of a synaptic response has been demonstrated in other systems (e.g., Kuno et al., 1971; Korn et al., 1981; Grantyn et al., 1984). A method commonly used to establish this relationship is quantal analysis of neurotransmitter release (Korn et al., 1982; Shepherd and Murphey, 1986; Hamon et al., 1990; Laurent and Sivaramakrishnan, 1992). Our working hypothesis predicts that binomial $n$, which is related to the number of synaptic contacts, would decrease during APR's regression. Similar information could potentially be gained by counting the number of synaptic contacts between individual PH afferents and APR using andtomical methods (e.g., Shepherd and Murphey, 1986; Blagburn and Thompson, 1990). Although our hypothesis relating the number of synaptic contacts to EPSP amplitude was formulated to account for developmental changes in EPSP amplitude, it may also account for the relationship between $\mathrm{PH}$ position and EPSP amplitude within a developmental stage (Figs. 5, 6); the somatotopic arrangement of $\mathrm{PH}$ afferent arbors within the CNS (Peterson and Weeks, 1988) may influence the number of synaptic contacts that an affercnt forms with APR. Gradients in synaptic strength related to the segmental or positional location of presynaptic neurons have also been described in other systems (Bastiani and Mulloney, 1988; Hamon et al., 1990).

All recordings of unitary EPSPs on day $\mathrm{PO}$ in this study were made in heterochronic mosaic pupae with a retained larval proleg, thereby permitting individual PH sockets to be stimulated. Jacobs and Weeks (1990) showed previously that retained PH afferents maintain their larval anatomy in the pupal CNS, and other studies using heterochronic mosaic pupae with retained larval afferents have found that the synaptic effects of the af- 
ferents are consistent with their morphology and developmental stage (Levine et al., 1986; Levine, 1989). In interpreting our data, we assumed that PH afferents in heterochronic mosaic pupae retained their larval physiological propertics (c.g., ncurotransmitter release characteristics), just as they retained their larval anatomy. However, this important assumption has not been tested directly. Nevertheless, the most parsimonious interpretation of the existing data is that developmental changes in unitary EPSPs observed in heterochronic mosaic pupae (Fig. 6) resulted from changes in the postsynaptic rather than presynaptic neurons; experiments utilizing APR (this study) and PPR (Jacubs and Weeks, 1990) both support the idea that changes in proleg motoneurons are sufficient to account for the developmental decrease in synaptic input from $\mathrm{PH}$ afferents.

Although the APRs in segments A3 and A6 had different fates after pupation (survival or death, respectively), they did not differ in dendritic regression (Fig. 1), intrinsic electrical properties (Table 1), or the developmental decrease in cEPSP amplitude (Fig. 2). This was the case even on day P0, less than $24 \mathrm{hr}$ before the APRs in segment A6 would die (Weeks et al., 1992). Previous studies of PPR demonstrated that dendritic regression and programmed death are independent developmental events with different hormonal sensitivities; for example, PPR's regression and death can be dissociated by manipulating prepupal ecdysteroid levels or interfering with protein synthesis (Weeks, 1987; Weeks et al., 1992, 1993). The present finding that APRs in different segments regressed similarly, regardless of whether they were fated to live or die (Fig. 1), further supports the idea that regression and death are independently regulated. It is not known how the segment-specific pattern of APR fate is controlled, but ablation of pre- or postsynaptic partners of APR has not been found to alter its fate (Weeks and Davidson, 1994; J. L. Lubischer and J. C. Weeks, unpublished data).

The extent of the $\mathrm{PH}$ afferent termination field decreased between days $\mathrm{L} 3$ and $\mathrm{PO}$ (Fig. $8 \mathrm{~A}$ ), which could have resulted from the death and/or regression of individual afferents. A previous study found that some PH afferents die by day P0 (Jacobs and Weeks, 1990), and morphometric analysis of the posterior projection of an $\alpha$-PH afferent (Figs. 8,9 ) in this study suggested that regression did not occur. The same morphometric technique was adequate to resolve changes in the axonal arbors of other mechanosensory neurons during the larval-pupal transformation (Levine et al., 1985; Levinc 1989). If the structure of the $\alpha$-PII arbor in the posterior ganglion is representative of $\mathrm{PH}$ afferent anatomy in the segment of origin, then the decrease in the size of the central termination field of the $\mathrm{PH}$ afferent population during normal development (Fig. 8A) must result only from the death of PH afferents. The $\alpha-P H$ produced an EPSP in APRs in the posterior ganglion on day L3 (Fig. 9B), but for technical reasons we could not test this connection on day $\mathrm{P} 0$. However, we expect that the monosynaptic EPSP would have been smaller or absent, due to APR's regression. Although some $\mathrm{PH}$ afferents normally die by day $\mathrm{P} 0$, the amplitude of the cEPSP produced in APR (Fig. 2) and PPR (Jacobs and Weeks, 1990) by $\mathrm{VN}_{\mathrm{AAhr} 3}$ stimulation decreased to a similar extent in normal hemisegments (in which some afferents have died) and heterochronic mosaic hemisegments (in which most afferents are preserved). This finding suggests that, during normal development, the role of afferent death in the developmental decrease in cEPSP amplitude is overshadowed by changes in the proleg motoneurons.

Although steroid hormones have dramatic effects on neuronal structure and behavior in many animals, the causal links between neuronal and behavioral changes are poorly understood. We have addressed this issue using electrophysiological techniques applied to identified neurons with known behavioral functions in Manduca. Our finding that dendritic regression of proleg motoneurons at the onset of metamorphosis is associated with the weakening of specific monosynaptic sensory inputs and the loss of a larval-specific behavior has parallels in other species. For instance, in rodents, cyclical changes in gonadal steroid levels in males of seasonally breeding species, or during the estrus cycle in females, are associated with growth and regression of dendrites in specific neuronal populations, and the concomitant waxing and waning of sexual behaviors (e.g., Forger and Breedlove, 1987; Woolley et al., 1990). It is likely that the types of synaptic alterations we have observed in Manduca accompany steroid-induced neuroanatomical changes in other systems. Furthermore, the finding that steroid hormone receptors in insects and vertebrates belong to the same receptor superfamily (Koelle et al., 1991) suggests that molecular similarities may exist between species, as well.

\section{References}

Bacon JP, Altman JS (1977) A silver intensification method for cobalt filled neurons in whole mount preparations. Brain Res 138:359-363.

Bastiani MJ, Mulloney B (1988) The central projections of the stretch receptor neurons of crayfish: segmental gradients of synaptic probability and strength. J Neurosci 8:1264-1272.

Bell RA, Joachim FA (1976) Techniques for rearing laboratory colonies of tobacco hornworms and pink bollworms. Ann Entomol Soc Am 69:365-373.

Berry MS, Pentreath VW (1976) Criteria for distinguishing between monosynaptic and polysynaptic transmission. Brain Res 105:1-20.

Blagburn JM, Thompson KSJ (1990) Specificity of filiform hair afferent synapses onto giant interneurons in Periplaneta americana: anatomy is not a sufficient determinant. J Comp Neurol 302:255-271.

Bollenbacher WE (1988) The interendocrine regulation of larval-pupal development in the tobacco hornworm, Manduca sexta: a model. J Insect Physiol 34:941-947.

Bollenbacher WE, Smith SL, Goodman W, Gilbert WI (1981) Ecdysteroid titer during the larval-pupal-adult development of the tobacco hornworm, Manduca sexta. Gen Comp Endocrinol 44:302-306.

Breedlove SM (1992) Sexual dimorphism in the vertebrate nervous system. J Neurosci 12:4133 4142.

Forger NG, Breedlove SM (1987) Seasonal variation in mammalian striated muscle mass and motoneuron morphology. J Neurobiol 18 : $155-165$.

Grantyn R, Shapovalov AI, Shiriaev BI (1984) Relation between structural and release parameters at the frog sensory-motor synapse. $J$ Physiol (Lond) 349:459-474.

Hamon A, Guillet JC, Callec JJ (1990) A gradient of synaptic efficacy and its presynaptic basis in the cercal system of the cockroach. J Comp Physiol [A] 167:363-376.

Jack JJB, Noble D, Tsien RW (1988) Electric current flow in excitable cells. Oxford: Clarendon.

Jacobs GA, Weeks JC (1990) Postsynaptic changes at a sensory-tomotoneuron synapse contribute to the developmental loss of a reflex behavior during insect metamorphosis. J Neurosci 10:1341-1356.

Koelle MR, Talbot, WS, Segraves WA, Bender WA, Cherbas P, Hogness DS (1991) The Drosophila EcR gene encodes an ecdysone receptor, a new member of the steroid receptor superfamily. Cell 67:59-78.

Korn H, Triller A, Mallet A, Faber DS (1981) Fluctuating responses at a central synapse: $n$ of binomial fit predicts number of stained presynaptic boutons. Science 213:898-901.

Korn H, Mallet A, Triller A, Faber DS (1982) Transmission at a central inhibitory synapse. II. Quantal description of release, with a physical correlate for binomial $n$. J Neurophysiol 48:679-707.

Kuno M, Turkanis SA, Weakly JM (1971) Correlation between nerve terminal size and transmitter release at the neuromuscular junction of the frog. J Physiol (Lond) 213:545-556.

Laurent G, Sivaramakrishnan A (1992) Single local interneurons in the locust make central synapses with different properties of transmitter release on distinct postsynaptic neurons. J Neurosci 12:2370-2380. 
Levine RB (1989) Expansion of the central arborizations of persistent sensory neurons during insect metamorphosis: the role of the steroid hormone, 20-hydroxyecdysone. J Neurosci 9:1045-1054.

Levine RB, Truman JW (1982) Metamorphosis of the nervous system: changes in the morphology and the synaptic interactions of identified cells. Nature 299:250-252.

Levine RB, Weeks JC (1989) Reorganization of neural circuits and behavior during insect metamorphosis. In: Perspectives on neural systems and behavior (Carew TJ, Kelley D, eds). New York: Liss.

Levine RB, Pak C, Linn D (1985) The structure, function and metamorphic reorganization of somatotopically projecting sensory neurons in Manduca sexta larvae. J Comp Physiol [A] 157:1-13.

Levine RB, Truman JW, Linn D, Bate CM (1986) Endocrine regulation of the form and function of axonal arbors during insect metamorphosis. J Neurosci 6:293-299.

McEwen BS (1991) Our changing ideas about steroid effects on an ever-changing brain. Semin Neurosci 3:497-507.

Miles CI, Weeks JC (1991) Developmental attenuation of the pre-ecdysis motor pattern in the tobacco hornworm, Manduca sexta. J Comp Physiol [A] 168:179-190.

Peterson BA, Weeks JC (1988) Somatotopic mapping of sensory neurons innervating mechanosensory hairs on the larval prolegs of Manduca sexta. J Comp Neurol 275:128-144.

Rall W, Burke RE, Smith TG, Nelson PG, Frank K (1967) Dendritic location of synapses and possible mechanisms for the monosynaptic EPSP in motoneurons. J Neurophysiol 30:1169-1193.

Riddiford LM (1985) Hormone action at the cellular level. In: Comprehensive insect physiology, biochemistry, and pharmacology, Vol 8 (Kerkut GA, Gilbert LI, eds), pp 37-84. New York: Pergamon.

Sandstrom DJ (1993) Respecification of identified larval muscles, motoneurons and interneurons to pupal functions in Manduca sexta. $\mathrm{PhD}$ thesis, University of California, Berkeley.

Sandstrom DJ, Weeks JC (1991) Segment-specific fates of proleg muscles and motoneurons during metamorphosis in Manduca sexta. Soc Neurosci Abstr 17:1227.

Shepherd D, Murphey RK (1986) Competition regulates the efticacy of an identified synapse in crickets. J Neurosci 6:3152-3160.

Streichert LC, Sandstrom DJ, Weeks JC (1992) Segment-specific fate of homologous motoneurons in Manduca sexta is uncorrelated with developmental changes in motoneuron anatomy and physiology. Soc Neurosci Abstr 18:1413.

Trimmer BA, Weeks JC (1989) Effects of nicotinic and muscarinic agents on an identified motoneurone and its direct afferent inputs in larval Manduca sexta. J Exp Biol 144:303-337.

Trimmer BA, Weeks JC (1991) Activity-dependent induction of facil- itation, depression, and post-tetanic potentiation at an insect central synapse. J Comp Physiol [A] 168:27-43.

Trimmer BA, Weeks JC (1993) Muscarinic acetylcholine receptors modulate the excitability of an identified insect motoneuron. J Neurophysiol 69:1821-1836.

Weeks JC (1987) Time course of hormonal independence for developmental events in neurons and other cell types during insect metamorphosis. Dev Biol 124:163-176.

Weeks JC, Davidson SK (1994) Influence of interganglionic interactions on steroid-mediated dendritic reorganization and death of proleg motor neurons during metamorphosis in Manduca sexta. J Neurobiol 25:535-554.

Weeks JC, Frnst-Utzschneider K (1989) Respecification of larval proleg motoneurons during metamorphosis of the tobacco hornworm, Manduca sexta: segmental dependence and hormonal regulation, J Neurobiol 20:569-592.

Weeks JC, Jacobs GA (1987) A reflex behavior mediated by monosynaptic connections between hair afferents and motoneurons in the larval tobacco hornworm, Manduca sexta. J Comp Physiol [A] 160: 315-329.

Weeks JC, Levine RB (1992) Endocrine influences on the postembryonic fates of identified neurons during insect metamorphosis. In: Determinants of neuronal identity (Shankland M, Macagno E, eds), pp 293-322. San Diego: Academic.

Weeks JC, Truman JW (1984) Neural organization of peptide-activated ecdysis behaviors during the metamorphosis of Manduca sexta. II. Retention of the proleg motor pattern despite loss of the prolegs at pupation. J Comp Physiol 155:423-433.

Weeks JC, Truman JW (1985) Independent steroid control of the fates of motoneurons and their muscles during insect metamorphosis. $\mathrm{J}$ Neurosci 5:2290-2300.

Weeks JC, Truman JW (1986) Hormonally mediated reprogramming of muscles and motoneurons during the larval-pupal transfonmation of the tobacco hornworm, Manduca sexta. J Exp Biol 125:1-13.

Weeks JC, Roberts WM, Trimble DL (1992) Hormonal regulation and segmental specificity of motoneuron phenotype during metamorphosis of the tobacco hornworm, Manduca sexta. Dev Biol 149:185196.

Weeks JC, Davidson SK, Debu BHG (1993) Effects of a protein synthesis inhibitor on the hormonally-mediated regression and death of motoneurons in the tobacco hornworm, Manduca sexta. J Neurobiol 24:125-140.

Woolley CS, Gould E, Frankfurt M, McEwen BS (1990) Naturally occurring fluctuations in dendritic spine density on adult hippocampal pyramidal neurons. J Neurosci 10:4035-4039. 